\title{
Solubility of oxygen in $\boldsymbol{n}$-hexane and in $\boldsymbol{n}$-perfluorohexane. Experimental determination and prediction by molecular simulation
}

\author{
A. M. A. Dias, R. P. Bonifácio, I. M. Marrucho, $\dagger$ A. A. H. Pádua and M. F. Costa Gomes* \\ Laboratoire de Thermodynamique des Solutions et des Polymères, CNRS UMR 6003, \\ Université Blaise Pascal Clermont-Ferrand, 63177 Aubière, France. \\ E-mail: margarida.c.gomes@univ-bpclermont.fr
}

Received 31st July 2002, Accepted 28th November 2002

First published as an Advance Article on the web 17th December 2002

\begin{abstract}
The solubility of oxygen in $n$-hexane and in $n$-perfluorohexane was determined experimentally and calculated by computer simulation. A precision apparatus based on a saturation method at constant pressure was used to measure the solubility at temperatures from 288 to $313 \mathrm{~K}$ and close to atmospheric pressure. Henry's law coefficients, $H_{2,1}\left(T, p_{1}^{\text {sat }}\right)$, were obtained from the experimental data and their temperature dependence was represented by appropriate correlations. The precision of the results was characterised by average deviations of $H_{2,1}$ from these smoothing equations and is of $\pm 0.5 \%$ and $\pm 0.8 \%$ for oxygen in $n$-hexane and in $n$-perfluorohexane, respectively. From the temperature variation of the Henry's law coefficients, partial molar solvation quantities such as the variation of the Gibbs energy, enthalpy and entropy were derived. Molecular dynamics simulations with all-atom force fields, associated with Widom's test particle insertion method, were used to calculate the residual chemical potential of oxygen in the two solvents studied leading to Henry's law coefficients which were then compared to the experimental values. The difference between oxygen solubility in the two solvents was interpreted on the basis of solute-solvent interactions and structural properties such as solute-solvent radial distribution functions.
\end{abstract}

\section{Introduction}

Compared to hydrocarbons, fluorinated alkanes exhibit very different physical and chemical properties. ${ }^{1}$ For example, under the same conditions of temperature and pressure, and for molecules containing the same number of carbon atoms, fluorocarbon gases are less soluble than the equivalent hydrocarbon when dissolved in the same non-polar non-fluorinated solvent. Another important experimental generalization is that a fluorocarbon solvent will dissolve a larger amount of a particular gas than the equivalent hydrocarbon. These differences have been interpreted by describing gas solubility by a twostage process where the first step would consist on the formation of a large enough cavity within the liquid solvent to accommodate the solute molecule, and the second step would consist on the insertion of the solute within the solvent cavity. The large solubility of gases in the fluorocarbons is explained by the relative ease of cavity formation in these liquids. ${ }^{1}$ Gas solubility data can thus provide important information about the properties and structure of solutions. ${ }^{2}$ Furthermore, the interpretation of the solubility of gases in liquids and the study of the molecular structure of the resulting solutions using statistical mechanics calculations is feasible at present. Molecular simulations can be carried out using available rigorous intermolecular potential models allowing quantitative predictions of the solubility and of the derived thermodynamic properties associated with the dissolution process.

The peculiar physico-chemical properties of fluoro compounds also contribute to their recent applications in numerous fields. In particular, these substances are increasingly being used as substitutes for chlorinated solvents, as effective surfactants in supercritical solvents ${ }^{3}$ such as supercritical car-

$\dagger$ Present address: Department of Chemistry, University of Aveiro, Campus de Santiago, 3810-193 Aveiro, Portugal. bon dioxide, as environmental probes to determine the exchanges between the atmosphere and natural waters ${ }^{1}$ and in numerous biomedical applications ${ }^{4}$ (as artificial blood substitutes, gas-carriers in eye surgery or as ultrasound contrast agents).

The present work deals, on the one hand, with the precise experimental determination of the solubility of oxygen in liquid $n$-hexane and in liquid $n$-perfluorohexane, in a sufficiently large temperature range to allow the calculation of the thermodynamic quantities associated with the dissolution and to provide experimental information about the unlike solute-solvent molecular interactions. On the other hand, free energy calculations by molecular simulation are used both to test molecular interaction models, to calculate Henry's law coefficients in an extended range of thermodynamic conditions and to access to microscopic details such as the structure of the solutions, which are otherwise not easily observable. These two approaches may contribute to explain the differences encountered in the oxygen solubilities in the alkane and in the perfluoroalkane studied, by providing a better understanding of the molecular mechanisms governing the solubility phenomena.

\section{Experimental section}

\section{Materials}

Measurements were performed using $n$-hexane from Fluka with a stated purity greater than $99.5 \%$ and $n$-perfluorohexane from ABCR with a stated purity of $95 \%$ and from Acros (Fluorinert Liquid FC72). The oxygen used was from AGA/ Linde Gas with $99.999 \% \mathrm{~mol} / \mathrm{mol}$ minimum stated purity. Both the solvents and the gas were used without further purification.

Phys. Chem. Chem. Phys., 2003, 5, 543-549 


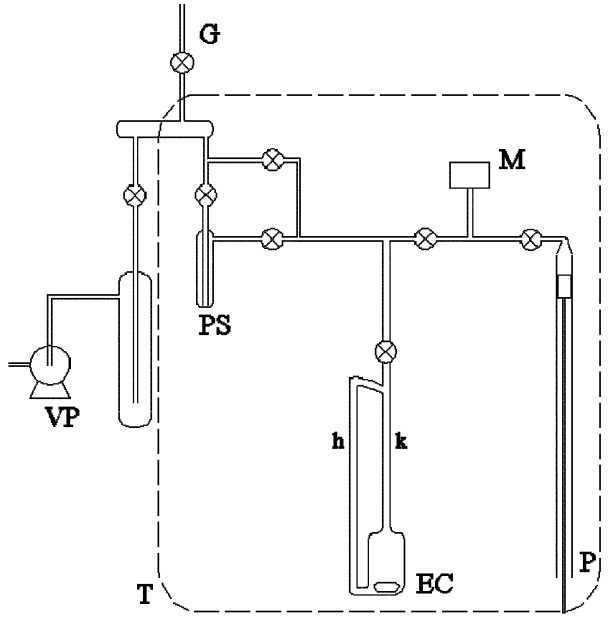

Fig. 1 Schematic drawing of the apparatus: VP, vacuum pump; G, gas inlet; PS, pre-saturator; EC, equilibrium cell with capillaries $h$ and $\mathrm{k} ; \mathrm{M}$, manometer; $\mathrm{P}$, piston; $\mathrm{T}$, air thermostat.

\section{Apparatus and operation}

The technique employed in this work makes use of the saturation method originally developed by Ben-Naim and Baer where the solubility is determined by measuring the quantity of gas dissolved in an accurately known volume of solvent at constant pressure and temperature. The experimental arrangement is mercury-free and similar to that extensively used by Battino and co-workers. ${ }^{6}$

The experimental apparatus is schematically represented in Fig. 1. It is housed in a large air thermostat ( $T$ in Fig. 1) where the temperature is maintained constant to within $\pm 0.01 \mathrm{~K}$ by means of a PID controller. The temperature is measured with a resolution of $0.01 \mathrm{~K}$ with a previously calibrated $25 \Omega$ platinum resistance thermometer.

The pure solvent is initially degassed by successive melting/ freezing cycles while vacuum pumping non-condensable gases. Up to eight cycles were necessary to degas the $n$-hexane and the $n$-perfluorohexane used as solvents. The degassed solvent is then transferred, under its own vapour pressure, to the dissolution cell (EC in Fig. 1). The saturation cell was built following the design of Carnicer et $a l^{7}$ and has an accurately known volume of approximately $6 \mathrm{~cm}^{3}\left(5.788 \pm 0.002 \mathrm{~cm}^{3}\right)$. The calibration of volume of the equilibrium cell was performed by relating this quantity with the height of the liquid in the capillaries $\mathrm{h}$ and $\mathrm{k}$ (see Fig. 1) at the different temperatures studied.

The solute gas is introduced in the apparatus through the saturation chamber indicated as PS in Fig. 1. The dissolution process is initiated by opening the stopcock just above the capillary tubes and by turning on the liquid circulation. The solvent is then forced up into capillary arm $h$ and descends through capillary arm $\mathrm{k}$. The volume of gas dissolved is determined by the displacement of the piston P (see Fig. 1) necessary to maintain a constant pressure in the system. The close-fitting piston is constituted of a Teflon seal backed up by O-rings, through a Fisher and Porter 5/8 inch precision bore tube (with a diameter of $15.875 \pm 0.008 \mathrm{~mm}$ at $0{ }^{\circ} \mathrm{C}$ ). The displacement of the piston is determined with a cathetometer Edouard Bounty and Cie with a precision of \pm 0.002 $\mathrm{cm}$. The pressure is read in a precision manometer from Drück, Model DPI 262 (1.4 bar F.S.) calibrated with a precision of \pm 0.001 bar.

\section{Data reduction}

The Ostwald coefficient, $L_{2,1}(T, p)$, is a very convenient way of expressing the solubility of a gas in a liquid, ${ }^{8}$ several definitions of which are currently used. However, in the one considered as thermodynamically more rigorous, $L_{2,1}(T, p)$ is expressed as: ${ }^{6}$

$$
L_{2,1}(T, p)=\left(\frac{C_{2}^{\mathrm{L}}}{C_{2}^{\mathrm{V}}}\right)_{\text {eq. }}
$$

where $C_{2}^{\mathrm{L}}=n_{2}^{\mathrm{L}} / V^{\mathrm{L}}$ and $C_{2}^{\mathrm{V}}=n_{2}^{\mathrm{V}} / V^{\mathrm{V}}$ are the amount of substance concentrations of the liquid solution (with molar volume $V^{\mathrm{L}}$ ) and of the vapour phase in equilibrium (with molar volume $V^{\mathrm{V}}$ ) at $T$ and $p$, respectively. From this definition, the experimentally measured quantities can also be related to the Ostwald coefficient, eqn. (1) being equivalent to

$$
L_{2,1}(T, p)=\frac{v^{\mathrm{V}}}{v^{\mathrm{L}}}
$$

where $v^{\mathrm{V}}$ is the volume of gas dissolved and $v^{\mathrm{L}}$ the total volume of the liquid solution after equilibrium is reached.

The molar fraction of component 2 (taken here to be the gaseous solute) in the liquid solution can be directly related to the Ostwald coefficient:

$$
x_{2}=\frac{L_{2,1}(T, p) p_{2} V^{\mathrm{L}}(T, p)}{Z_{12} R T}
$$

where $p_{2}$ is the partial pressure of the solute and $V^{\mathrm{L}}(T, p)$ the molar volume of the liquid solution (which can be taken as the orthobaric molar volume of the pure solvent, $V_{1}^{\mathrm{o}}$, in the conditions studied in this work). $Z_{12}$ is the compressibility factor of the solution:

$$
Z_{12}=1+\frac{p}{R T}\left(y_{1} B_{11}+y_{2} B_{22}+y_{1} y_{2} \delta_{12}\right)
$$

where $B_{11}$ and $B_{22}$ are the second virial coefficients for the pure solvent and the pure solute, respectively and $\delta_{12}=2 B_{12}-B_{11}-B_{22}$ where $B_{12}$ is the solute-solvent cross second virial coefficient.

The solubility can also be expressed in terms of the Henry's law coefficient which can be defined as: ${ }^{9}$

$$
H_{2,1}(T, p)=\lim _{x_{2} \rightarrow 0}\left[\frac{f_{2}\left(p, T, x_{2}\right)}{x_{2}}\right]
$$

where $f_{2}\left(p, T, x_{2}\right)=\phi_{2}(T, p) p_{2}$ is the fugacity of component 2 in the solution, $\phi_{2}(T, p)$ being the fugacity coefficient, calculated in the usual way. ${ }^{9}$ The dependence of the Henry's law coefficient with pressure can be expressed as: ${ }^{10}$

$$
H_{2,1}(T, p)=H_{2,1}\left(T, p_{1}^{\mathrm{sat}}\right) \exp \int_{p_{1}^{\text {sat }}}^{p} \frac{V_{2}^{\infty}(T, p)}{R T} \mathrm{~d} p
$$

where $V_{2}^{\infty}(T, p)$ is the partial molar volume of the solute at infinite dilution and $p_{1}^{\text {sat }}$ is the vapour pressure of the pure solvent.

The Henry's law coefficient can then be related with the experimentally determined Ostwald coefficient

$$
\begin{aligned}
H_{2,1}\left(T, p_{1}^{\mathrm{sat}}\right)= & \frac{R T Z_{12}(T, p) \phi_{2}(T, p)}{V_{1}^{\mathrm{o}}(T) L_{2,1}(T, p)} \\
& \times \exp \left[\frac{-V_{2}^{\infty}(T)\left(p-p_{1}^{\mathrm{sat}}\right)}{R T}\right]
\end{aligned}
$$

where the molar volume of the solution was approximated by the orthobaric molar volume of the pure solvent (see eqn. (3)) and the pressure dependence of both $L_{1,2}(T, p)$ and $V_{2}^{\infty}(T, p)$ was considered negligible.

When expressed as a molar fraction, the solubility is normally given at a constant value of the partial pressure of the gas (for example at $p_{2}=101325 \mathrm{~Pa}$ ). It is nevertheless necessary to determine the molar fractions in the vapour phase in equilibrium with the liquid solution, $y_{i}$, in order to calculate the product $\phi_{2} Z_{12}$ (normally close to unity). This quantity is obtained iteratively making use of the phase equilibrium 
condition for component 1 (the liquid solvent):

$$
\phi_{1}(T, p) y_{1} p=\gamma_{1}(T, p) x_{1} f_{1}^{\mathrm{o}}
$$

where $\phi_{1}$ is the fugacity coefficient of component $1, \gamma_{1}$ its activity coefficient (considered to be unity in the thermodynamic conditions of this work), $p$ the equilibrium pressure, $f_{1}^{\text {o }}$ the standard state fugacity and $x_{1}$ and $y_{1}$ the molar fractions in the liquid solution and in the gaseous phase in equilibrium with it, respectively.

Eqn. (8) can be rewritten $a^{6}$ (the dependences of the different functions are omitted for the sake of simplicity):

$$
y_{1}=\left(1-x_{2}\right)\left(\frac{p_{1}^{\mathrm{sat}}}{p}\right)\left(\frac{\phi_{1}^{\mathrm{sat}}}{\phi_{1}^{\mathrm{o}}}\right) \exp \left[\frac{V_{1}^{\mathrm{o}}\left(p-p_{1}^{\mathrm{sat}}\right)}{R T}\right]
$$

The fugacity coefficient and the standard state fugacity of component 1 are calculated in the usual way. ${ }^{9}$

\section{Experimental results}

Temperatures are reported on the ITS-90 scale. The relative atomic masses used are the ones recommended by IUPAC ${ }^{11}$ and the considered value for the gas constant was 8.31451 J $\mathrm{mol}^{-1} \mathrm{~K}^{-1} \cdot{ }^{12}$

The vapour pressure of the pure solvents was taken from the compilation of Ruzicka and Majer ${ }^{13}$ for $n$-hexane and from the work of Stiles and $\mathrm{Cady}^{14}$ for $n$-perfluorohexane. The second virial coefficients were obtained from the compilation of Dymond and Smith ${ }^{15}$ except for $n$-hexane for which the values recommended by Dymond et al. ${ }^{16}$ were used. The cross virial coefficients were estimated for both solutions, using the correlation by Tsonopoulos. ${ }^{17}$

The partial molar volumes of oxygen dissolved in $n$-hexane and of oxygen dissolved in $n$-perfluorohexane were calculated, as a function of temperature, using the method described by Tiepel and Gubbins ${ }^{18}$ which is based on a first-order perturbation theory. For oxygen in hexane, the partial molar volume can be expressed, as a function of temperature between 288 and $313 \mathrm{~K}$ by:

$$
\ln \left(V_{2}^{\infty} / \mathrm{cm}^{3} \mathrm{~mol}^{-1}\right)=3.511+2.39 \times 10^{-3} t
$$

where $t=T / \mathrm{K}-273.15$. The value obtained at $298.15 \mathrm{~K}$ was compared to the experimental result of $\mathrm{Ng}$ and Walkley ${ }^{19}$ and a negative deviation of $20.2 \mathrm{~cm}^{3} \mathrm{~mol}^{-1}$ (approximately $35 \%$ ) was found. Although this deviation affects negligibly the values of the Henry's law coefficient (a $50 \%$ change in $V_{2}^{\infty}$ causes a deviation as small as $0.15 \%$ in $H_{2,1}$ ), the experimental value was considered for the calculations and the temperature dependence was not taken into account.

For the partial molar volume at infinite dilution of oxygen in perfluorohexane, no experimental values were found in the literature. The values obtained by the Tiepel and Gubbins method can be expressed, as a function of temperature between 288 and $313 \mathrm{~K}$ by the relation:

$$
\ln \left(V_{2}^{\infty} / \mathrm{cm}^{3} \mathrm{~mol}^{-1}\right)=3.676+3.37 \times 10^{-3} t
$$

Table 1 shows the experimental values for the solubility of oxygen in the two liquids studied between 288 and $318 \mathrm{~K}$. The temperature and pressure of the measurements, the values for the Ostwald coefficients, the calculated mole fractions and Henry's law coefficients at the saturation pressures of the pure solvents, are reported.

From the several empirical correlations proposed in the literature to represent the temperature dependence of Henry's law coefficients, the one proposed by Krause and Benson ${ }^{20}$ was adopted in this work. The experimental values for $H_{2,1}\left(T, p_{1}^{\text {sat }}\right)$ were fit to a power series in $1 / T$ :

$$
\ln H_{2,1}\left(T, p_{1}^{\mathrm{sat}}\right)=\sum_{i=0}^{n} B_{i} T^{-i}
$$

Table 1 Experimental data for the solubility of oxygen in hexane and perfluorohexane between 288 and $313 \mathrm{~K}$ expressed as Ostwald coefficients, mole fraction solubilities at a solute partial pressure of $101325 \mathrm{~Pa}$ and Henry's law coefficients at the saturation pressure of the pure solvent

\begin{tabular}{lllll}
\hline$T / \mathrm{K}$ & $p / \mathrm{MPa}$ & $L_{2,1}(T, p)$ & $10^{3} x_{2}$ & $H_{2,1}(T, p)_{1}^{\text {sat }} / \mathrm{MPa}$ \\
\hline \multicolumn{5}{c}{$n-\mathrm{C}_{6} \mathrm{H}_{14}$} \\
288.89 & 0.0934 & 0.395 & 2.17 & 46.7 \\
293.20 & 0.0955 & 0.386 & 2.10 & 48.2 \\
298.10 & 0.0992 & 0.368 & 1.99 & 51.0 \\
298.51 & 0.0952 & 0.369 & 1.99 & 50.9 \\
298.86 & 0.0922 & 0.368 & 1.99 & 51.0 \\
303.43 & 0.0901 & 0.341 & 1.83 & 55.5 \\
307.84 & 0.0966 & 0.320 & 1.69 & 60.1 \\
312.44 & 0.0993 & 0.296 & 1.55 & 65.6 \\
\hline \multicolumn{5}{c}{$n-\mathrm{C}_{6} \mathrm{~F}_{14}$} \\
288.69 & 0.0962 & 0.592 & 4.94 & 20.5 \\
293.38 & 0.0962 & 0.567 & 4.68 & 21.6 \\
299.36 & 0.0986 & 0.517 & 4.23 & 23.9 \\
303.36 & 0.0772 & 0.461 & 3.75 & 26.9 \\
303.37 & 0.0651 & 0.466 & 3.79 & 26.6 \\
303.41 & 0.0665 & 0.460 & 3.75 & 27.0 \\
303.47 & 0.0967 & 0.463 & 3.76 & 26.9 \\
307.83 & 0.0979 & 0.423 & 3.42 & 29.6 \\
312.58 & 0.0960 & 0.366 & 2.94 & 34.5 \\
\hline
\end{tabular}

The coefficients $B_{i}$ as well as the average absolute deviations obtained for the Henry's law coefficients of oxygen in $n$-hexane and in $n$-perfluorohexane are listed in Table 2.

In order to evaluate the accuracy of the experimental technique used, the solubilities of oxygen in $n$-hexane obtained were compared to literature values. The results of this work for the Henry's law coefficient at $298.15 \mathrm{~K}$ do not deviate more than $1.0 \%$ from the values reported in the literature. ${ }^{21,22}$ The datum obtained at $313.15 \mathrm{~K}$ was compared to the sole value found in the literature, ${ }^{22}$ and it was observed that the Ostwald coefficient measured in this work is $1.5 \%$ higher, but this deviation lies within the uncertainty of $\pm 3 \%$ claimed by the authors. ${ }^{22}$ From the analysis of these data and after a careful study of the sources and order of magnitude of the systematic errors during our experiments, it is believed that the present values of solubility are accurate to within $1 \%$.

The average absolute deviations of the fits using eqn. (12) characterise the precision of the experimental results. ${ }^{23,24}$ The values for the solubility of oxygen in $n$-hexane, expressed as Henry's law coefficients, are precise to within $0.5 \%$ (see Table 2). For the case of the solubility of oxygen in perfluorohexane, also expressed as $H_{2,1}$, the results are precise to within $0.8 \%$ (see Table 2). This larger imprecision is likely due to the larger volatility of the fluorinated solvent (which renders experiments more difficult) and to the use of two different samples of perfluorohexane.

The solubility of oxygen in perfluorohexane, at approximately $303.15 \mathrm{~K}$, was determined at different pressures in order to check the validity of Henry's law.

Table 2 Coefficients for eqn. (12) and average absolute deviation (AAD) for the correlation of the experimental data

\begin{tabular}{llll}
\hline$B_{0}$ & $B_{1}$ & $B_{2}$ & AAD \\
\hline $3.8310 \times 10^{6}$ & $-2.6901 \times 10^{4} \mathrm{H}_{14}$ & $6.4874 \times 10^{1}$ & $0.53 \%$ \\
\hline$n-\mathrm{C}_{6} \mathrm{H}_{14}$ & & \\
$3.9986 \times 10^{6}$ & $-2.8629 \times 10^{4}$ & $6.8020 \times 10^{1}$ & $0.77 \%$ \\
\hline
\end{tabular}

Phys. Chem. Chem. Phys., 2003, 5, 543-549 
Table 3 Partial molar thermodynamic properties of solution for oxygen in hexane and in perfluorohexane. The values are calculated at several temperatures between 285 and $315 \mathrm{~K}$ and are relative to the ideal gas state at $101325 \mathrm{~Pa}$

\begin{tabular}{|c|c|c|c|}
\hline$T / \mathrm{K}$ & $\Delta G_{2}^{\mathrm{o}} / \mathrm{kJ} \mathrm{mol}^{-1}$ & $\Delta H_{2}^{\mathrm{o}}$ & $\Delta S_{2}^{\mathrm{o}}$ \\
\hline \multicolumn{4}{|c|}{$n-\mathrm{C}_{6} \mathrm{H}_{14}$} \\
\hline 285 & 14.51 & -0.1 & -0.5 \\
\hline 290 & 14.80 & -4.0 & -14 \\
\hline 295 & 15.16 & -7.7 & -26 \\
\hline 300 & 15.58 & -11.3 & -38 \\
\hline 305 & 16.05 & -14.8 & -49 \\
\hline 310 & 16.59 & -18.1 & -59 \\
\hline 315 & 17.17 & -21.4 & -68 \\
\hline \multicolumn{4}{|c|}{$n-\mathrm{C}_{6} \mathrm{~F}_{14}$} \\
\hline 285 & 12.49 & -4.7 & -17 \\
\hline 290 & 12.83 & -8.7 & -30 \\
\hline 295 & 13.23 & -12.6 & -43 \\
\hline 300 & 13.70 & -16.4 & -55 \\
\hline 305 & 14.23 & -20.0 & -66 \\
\hline 310 & 14.82 & -23.5 & -76 \\
\hline 315 & 15.47 & -26.9 & -86 \\
\hline
\end{tabular}

\section{Thermodynamic functions}

The exact expression for the change in the partial molar Gibbs energy when the solute is transferred, at temperature $T$, from the pure perfect gas at standard pressure to the infinitely dilute state in the solvent (standard Gibbs energy of solvation ${ }^{20}$ ) is

$$
\Delta G_{2}^{\mathrm{o}}\left(T, p_{1}^{\mathrm{sat}}\right)=R T \ln \left[\frac{H_{2,1}\left(T, p_{1}^{\mathrm{sat}}\right)}{p^{\mathrm{o}}}\right]
$$

where $p^{\circ}$ is the standard pressure. The difference in partial molar enthalpy between the two states can be obtained from the partial derivative of the Gibbs energy with respect to temperature at constant pressure. ${ }^{9}$ The enthalpy of solvation at temperature $T$ and at the pure solvent saturation pressure is: ${ }^{20}$

$$
\begin{aligned}
\Delta H_{2}^{\mathrm{o}}\left(T, p_{1}^{\mathrm{sat}}\right)= & -R T^{2}\left[\frac{\mathrm{d}}{\mathrm{d} T}\left(\frac{\ln H_{2,1}\left(T, p_{1}^{\mathrm{sat}}\right)}{p^{\mathrm{o}}}\right)\right. \\
& \left.-\frac{V_{2}^{\infty}(T)}{R T}\left(\frac{\mathrm{d} p_{1}^{\mathrm{sat}}(T)}{\mathrm{d} T}\right)\right]
\end{aligned}
$$

A similar treatment can be adopted to calculate the partial molar entropy at constant pressure. ${ }^{20}$ The values for the different partial molar thermodynamic functions of solvation of oxygen in $n$-hexane and $n$-perfluorohexane, calculated from the fitted Henry's law coefficients as given by eqn. (12), are listed in Table 3. The results are relative to the ideal gas state at $101325 \mathrm{~Pa}$. No data obtained by direct calorimetric determinations were found for comparison.

\section{Molecular simulation}

The solubility of oxygen in hexane and perfluorohexane was studied by computer simulation. Configurations of the pure solvents were generated by molecular dynamics and the residual chemical potential of the solute at infinite dilution, $\mu_{2}^{r}$, was calculated using the method of test particle insertion. The residual chemical potential of the solute at infinite dilution is the difference between the chemical potential of the solute in the solution and that of the solute in the pure ideal gas state at the same temperature and density, and is related to the standard Gibbs energy of solvation, defined in eqn. (13), by

$$
\Delta G_{2}^{\mathrm{o}}(T, p)=\mu_{2}^{r}(T, p)+R T \ln \frac{p^{\ominus}}{p^{\mathrm{o}}}
$$

where $p^{\ominus}=R T \rho_{1}$ is the pressure of the ideal gas phase at the density of the infinitely dilute solution, $\rho_{1}$.

\section{Intermolecular potential models}

Both solvents were represented by flexible force fields in which all atoms are considered explicitly. Parameters for the $n$-hexane and $n$-perfluorohexane molecules were taken from the OPLS-All Atom force field for alkanes ${ }^{25}$ and for perfluoroalkanes, ${ }^{26}$ respectively. This kind of molecular model ascribes to each atom an interaction site consisting of a Lennard-Jones function plus an electrostatic point charge. Within the molecules, flexible chemical bonds, valence angles and torsions (dihedral angles) are all specified using different potential energy functions. The OPLS-AA framework was chosen for the present application because it describes alkanes and perfluoroalkanes with equivalent levels of detail. A possible alternative could be based on united-atom models for alkanes ${ }^{27}$ and perfluoroalkanes, ${ }^{28}$ but our previous experience ${ }^{29}$ showed that the all-atom models perform better when predicting gas solubilities, in particular that of oxygen. The main deficiency of united-atom models is probably the absence of partial charges on individual atoms. The torsional energetics of the carbon skeletons, for example, can be represented correctly in both types of model.

Oxygen was also modelled by an intermolecular potential consisting of Lennard-Jones sites, in this case the rigid twocentre model of Miyano. ${ }^{30}$ The interactions between the gaseous solutes and the solvents were assumed to obey geometric combining rules for both Lennard-Jones parameters, $\sigma$ and $\varepsilon$, and therefore no specific unlike interaction terms were introduced.

\section{Simulation procedure}

The simulations of the pure liquid consisted of cubic boxes containing 200 molecules, and were carried out using the molecular dynamics package DL_POLY. ${ }^{31}$ The intermolecular potential parameters, including partial charges, and the intramolecular energy terms were retrieved from the OPLS-AA publications. ${ }^{25,26}$ The Lennard-Jones non-bonded interactions were considered explicitly up to a cutoff radius of $12 \AA$. Coulombic interactions were computed by Ewald summations ${ }^{32,33}$ over a number of reciprocal space vectors ranging from 7 to 9 , depending on the temperature and on the molecule in question. The width of the screening charge distribution was established by the parameter $\alpha=0.225 \AA^{-1}$. Flexibility was included for all types of intramolecular energy except for $\mathrm{C}-\mathrm{H}$ bonds, which were constrained to remain rigid at their equilibrium distance. This simplification allows the use of a time step of $1 \mathrm{fs}$ in molecular dynamics simulations while obeying conservation of energy in the $N V E$ ensemble (energy fluctuations within $1 \times 10^{-4}$ and no drift). After the necessary equilibrations, the solvents were simulated during 100 ps at constant pressure, temperature and amount of substance $(N p T)$, by means of Nosé-Hoover thermostats and barostats. The pressures were chosen slightly above the saturated vapour pressure, in order to guarantee sampling of the liquid phase. From each run 1000 configurations (snapshots) were stored at regular intervals.

The residual chemical potential of the solute gas was calculated using the test-particle insertion method. ${ }^{34}$ In each of the solvent snapshots previously stored, insertion of the solute molecule at random positions and orientations was tried 60000 times. For simulations at constant $N p T,{ }^{32}$

$$
\mu_{2}^{r}(T, p)=-k T \ln \frac{\left\langle V \exp \left(-u_{\mathrm{TP}} / k T\right)\right\rangle_{N p T}}{\langle V\rangle_{N p T}}
$$

where $u_{\mathrm{TP}}$ is the interaction energy of the test particle with a configuration of solvent molecules occupying volume $V$, and 
Table 4 Simulation results for oxygen in $n$-hexane and in $n$-perfluorohexane: chemical potential at infinite dilution, standard Gibbs energy of solvation and Henry's law coefficient

\begin{tabular}{llllll}
\hline$T / \mathrm{K}$ & $p / \mathrm{MPa}$ & $\rho_{1} / \mathrm{MPa}$ & $\mu_{2}^{r} / \mathrm{kJ} \mathrm{mol}^{-1}$ & $\Delta G_{2}^{\mathrm{o}} / \mathrm{kJ} \mathrm{mol}^{-1}$ & $H_{2,1} / \mathrm{MPa}$ \\
\hline \multicolumn{5}{c}{$n-\mathrm{C}_{6} \mathrm{H}_{14}$} \\
200 & 0.01 & 8.73 & 0.28 & 8.54 & 17.2 \\
250 & 0.01 & 8.16 & 1.18 & 11.8 & 29.9 \\
280 & 0.05 & 7.75 & 1.17 & 13.2 & 29.8 \\
300 & 0.05 & 7.53 & 1.46 & 14.5 & 33.7 \\
330 & 0.1 & 7.13 & 1.53 & 14.4 & 34.2 \\
350 & 0.2 & 6.89 & 1.66 & 17.1 & 35.5 \\
400 & 0.6 & 6.11 & 1.54 & 19.2 & 32.3 \\
450 & 1.5 & 5.08 & 1.23 & 20.8 & 26.4 \\
\hline & & & $n-\mathrm{C}_{6} \mathrm{H}_{14}$ & & \\
200 & 0.1 & 5.77 & 0.06 & 7.63 & 9.95 \\
250 & 0.1 & 5.37 & 0.89 & 10.7 & 17.1 \\
280 & 0.1 & 5.11 & 1.10 & 12.2 & 19.1 \\
300 & 0.1 & 4.91 & 1.07 & 13.0 & 18.8 \\
330 & 0.3 & 4.67 & 1.42 & 14.7 & 21.5 \\
350 & 0.3 & 4.50 & 1.53 & 15.7 & 22.2 \\
400 & 0.9 & 3.91 & 1.40 & 16.2 & 19.8 \\
\hline
\end{tabular}

$\langle\cdots\rangle_{N p T}$ denotes an isothermal-isobaric ensemble average over the stored configurations.

\section{Simulation results}

The values for the standard Gibbs energy of solvation and for the Henry's law coefficients of oxygen in $n$-hexane and in $n$ perfluorohexane calculated from simulation are listed in Table 4. The comparison to the experimental results, illustrated in Fig. 2, shows that the simulated values of the Gibbs energy of solvation (or Henry's law coefficient) are systematically higher, but the correct order of magnitude in the two solvents is predicted.

For oxygen in $n$-hexane, the difference between simulation and experiment is significant, but it is much smaller for $n$-perfluorohexane, in which case simulation provides a very good prediction of the solubility around $300 \mathrm{~K}$. However, it is surprising to observe that, for both solvents, the temperature dependence of the solubility exhibits markedly different behaviours when simulation is compared to experiment. The simulated Henry's law coefficients pass through a maximum in the temperature range studied, whereas the experimental results (in a much smaller temperature range) seem to present a steep

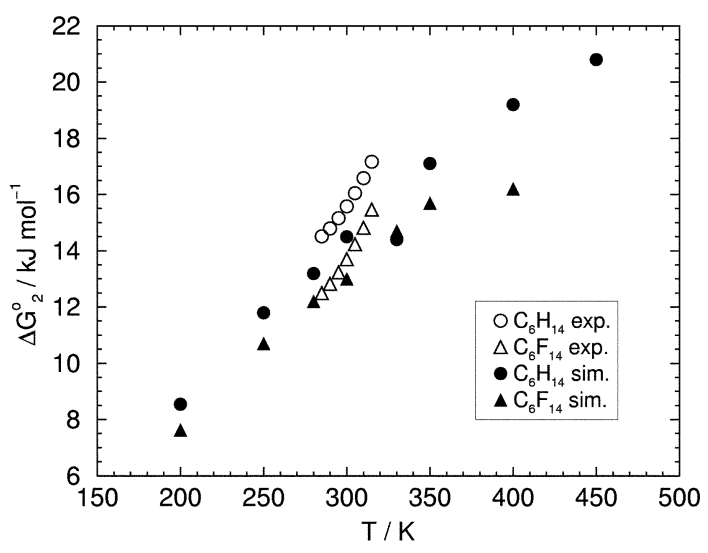

Fig. 2 Standard Gibbs energy of solvation for oxygen in $n$-hexane and in $n$-perfluorohexane. Experimental results for oxygen in $n$-hexane and in $n$-perfluorohexane (open circles and open triangles, respectively); simulation results for oxygen in $n$-hexane and $n$-perfluorohexane (solid circles and solid triangles, respectively). increase of this property with temperature. The two experimental sets are consistent and the two simulated sets are also consistent, but the trend of the simulated values fails to reproduce experiment. Unfortunately, the experimental temperature range is narrow and leaves some questions unanswered. Previous work on the solubility of xenon in the same solvents yielded results which are the opposite of the present ${ }^{35}$ the correct temperature dependence was predicted well from the start, but binary interaction parameters had to be introduced in order to superimpose the simulated values onto the experimental ones.

Part of the difference of solubilities can be explained by the interaction energies between oxygen and the two solvents. However, factors related to the structure of the liquid solvents seem to play an important role. The normal alkane molecule is more flexible than its perfluorocarbon counterpart, and thus the latter tends to adopt linear conformations more frequently in the liquid phase. The increased rigidity of the perfluorocarbon when compared to the analogous hydrocarbon at the same temperature probably means that larger cavities (at the molecular scale) are spontaneously present in the liquid perfluorocarbon than in the hydrocarbon. This certainly has a significant incidence on the solubility of small gaseous molecules.

Atom-atom radial distribution functions of oxygen in both solvents are plotted in Figs. 3 and 4. The larger molecular volume of the perfluoroalkane is evident from the "wavelength" of the oscillations in local density. In amplitude, these oscillations are also more marked in the perfluoroalkane, especially beyond the first maximum, a manifestation of a more ordered liquid structure, which we interpret as a result of the reduced flexibility of the perfluorocarbon molecules.
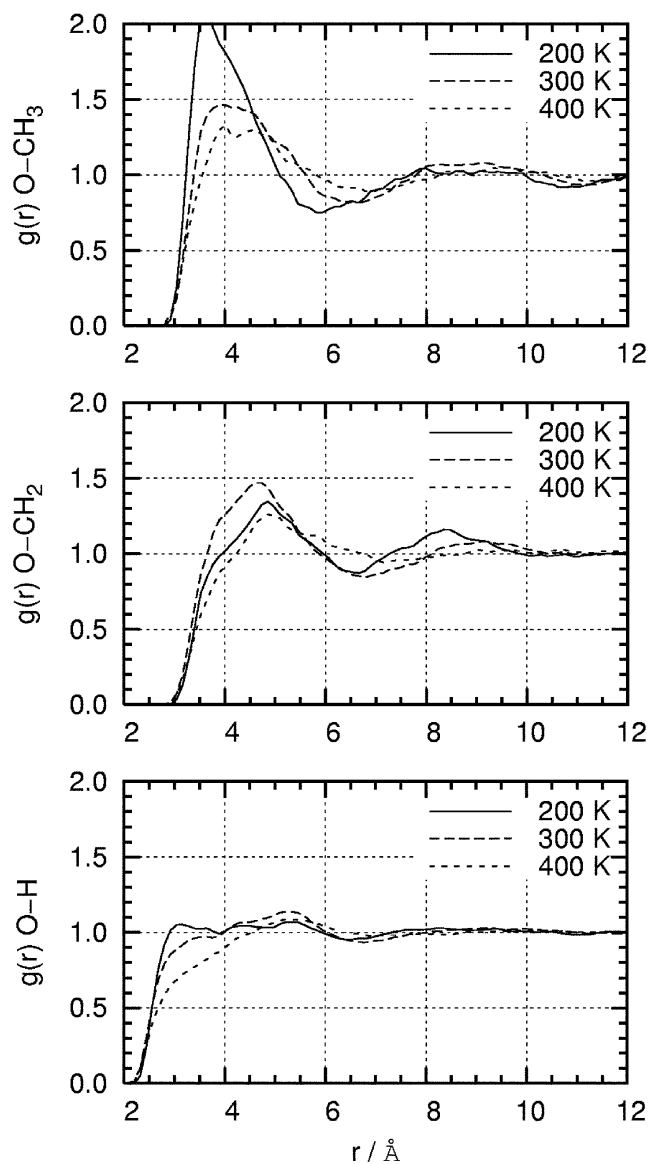

Fig. 3 Solute-solvent atom-atom radial distributions functions of oxygen in $n$-hexane. 

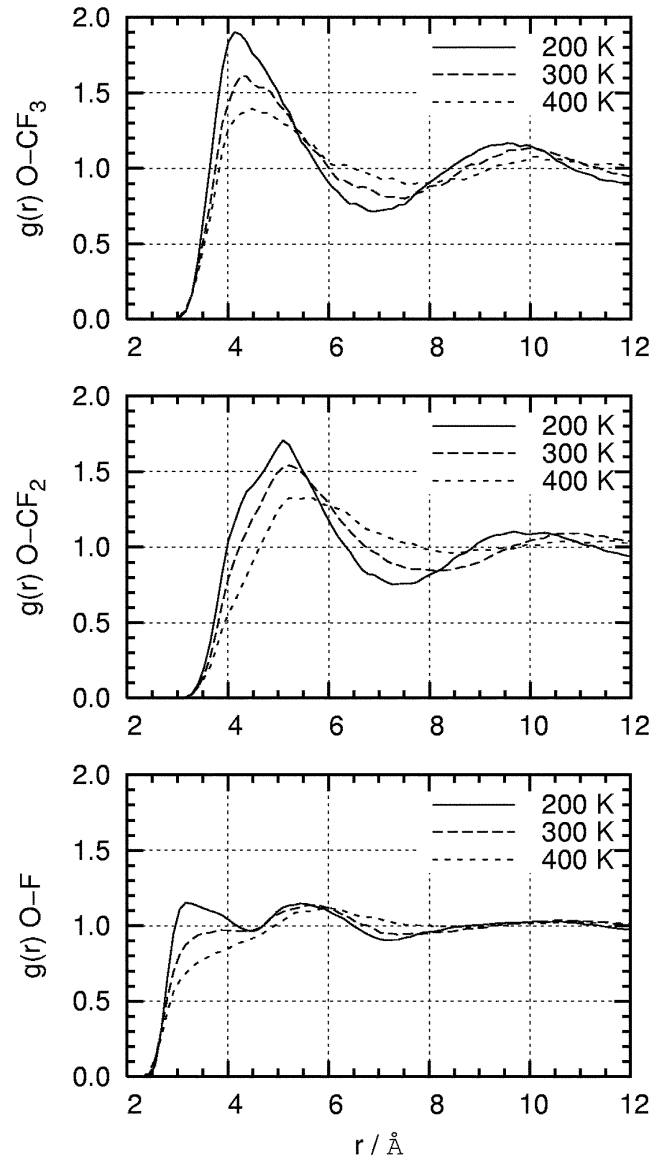

Fig. 4 Solute-solvent atom-atom radial distributions functions of oxygen in $n$-perfluorohexane.

\section{Conclusions}

The present paper reports original experimental results of the solubility of oxygen in $n$-hexane and $n$-perfluorohexane as a function of temperature from 288 to $313 \mathrm{~K}$. The temperature range studied allows the derivation of several thermodynamic quantities characteristic of the dissolution process that up to now had not been determined directly.

As expected, the fluorinated solvent dissolves a larger amount of oxygen than the equivalent hydrocarbon, the difference in solubilities being more important at the lower temperature studied. This behaviour is qualitatively reproduced by computer simulation which was employed as a predictive tool, using force fields taken from literature. It has been shown ${ }^{29}$ that the choice of all-atom force fields ${ }^{26}$ is pertinent as it provides better agreement with experimental data than do unitedatom models. ${ }^{28}$

The Henry's law coefficients, being a property at infinite dilution, depend on the solute-solvent interactions and not on those present in the pure solute. This aspect may contribute to improve interaction models for mixtures. The approximation consisting of adopting simple combining rules to take into account the solute-solvent interactions was used here although a quantitative prediction of the experimental results was not possible. It has been shown previously ${ }^{35}$ that whenever the behaviour of the Henry's law coefficient (or of the Gibbs energy of solvation) with temperature is reproduced by simulation, it is possible to improve the combining rules, making use of the experimental data. Quantitative predictions of Henry's law coefficients are then achieved.

The combined approach of the present work demonstrates that computer simulation can serve as a complement to experimental measurements allowing the extension of the ranges of conditions where data are available and permitting the access to molecular details concerning the dissolution process.

\section{Acknowledgements}

This work benefited from allocation of computer resources at the Institut du Développement et des Resources en Informatique Scientifique (IDRIS) du Centre National de la Recherche Scientifique, France. The authors are grateful to Fundação para a Ciência e Technologia for the grants awarded to R.P.B. and A.M.A.D. The collaboration between the French and Portuguese teams was supported by the Instituto para a Cooperação Cientifica e Tecnologica/French Embassy in Portugal cooperation program. The authors thank Dr S. Sarraute and $\mathrm{Mr}$ J. Deschamps for their participation in the preliminary stages of this work.

\section{References}

1 S. L. Swinton, Chemical Thermodynamics, The Chemical Society, 1978, vol. 2.

2 J. H. Hildebrand, J. M. Prausnitz and R. L. Scott, Regular and Related Solutions, Van Nostrand Reinhold Co., New York, 1970.

3 J. B. McClain, D. E. Betts, D. A. Canelas, E. T. Samulski, J. M. DeSimone, J. D. Londono, H. D. Cochran, G. D. Wignall, D. Chillura-Martino and R. Triolo, Science, 1996, 274, 2049.

4 J. G. Riess, Chem. Rev., 2001, 101, 2797.

5 A. Ben-Naim and S. Baer, Trans. Faraday Soc., 1963, 59, 2735.

6 T. Tominaga, R. Battino, H. K. Gorowara, R. D. Dixon and E. Wilhelm, J. Chem. Eng. Data, 1986, 31, 175.

7 J. Carnicer, F. Gibanel, J. S. Urieta and C. G. Losa, Rev. Acad Cienc. Exactas, Fis.-Quim. Nat. Zaragoza, 1979, 34, 115.

8 R. Battino, Fluid Phase Equilib., 1984, 15, 231.

9 J. M. Smith, H. C. V. Ness and M. M. Abbott, Introduction to Chemical Engineering Thermodynamics, McGraw-Hill, New York, 5th edn., 1996.

10 B. B. Benson and D. Krause, J. Solution Chem., 1989, 18, 803.

11 IUPAC, Pure Appl. Chem., 1986, 58, 1677.

12 IUPAC, Quantities, Units and Symbols in Physical Chemistry, Blackwell Scientific Publications, Oxford, 1988.

13 K. Ruzicka and V. Majer, J. Phys. Chem. Ref. Data, 1994, 23, 1.

14 V. E. Stiles and G. H. Cady, J. Am. Chem. Soc., 1952, 74, 3771.

15 J. H. Dymond and E. B. Smith, The Virial Coefficients of Pure Gases and Mixtures, Oxford University Press, Oxford, 1980.

16 J. H. Dymond, J. Cholinski, A. Szafranski and D. WyrzykowskaStankiewicz, Fluid Phase Equilib., 1986, 27, 1.

17 B. E. Poling, J. M. Prausnitz and J. P. O'Connell, The Properties of Gases and Liquids, McGraw-Hill, New York, 5th edn., 2001

18 E. W. Tiepel and K. E. Gubbins, Can. J. Chem. Eng., 1972, 50, 361 .

19 W. Y. Ng and J. Walkley, J. Phys. Chem., 1969, 73, 2274.

20 D. Krause and B. B. Benson, J. Solution Chem., 1989, 18, 823.

21 P. J. Hesse, R. Battino, P. Scharlin and E. Wilhelm, J. Chem. Eng. Data, 1996, 41, 195.

22 J. Makranczy, K. Megyery-Balog, L. Rusz and L. Patyi, Hung. J. Ind. Chem., 1976, 4, 269.

23 M. F. Costa Gomes and J. P. Grolier, Phys. Chem. Chem. Phys., 2001, 3, 1047

24 R. P. Bonifácio, A. A. H. Pádua and M. F. Costa Gomes, J. Phys Chem. B, 2001, 105, 8403

25 W. L. Jorgensen, D. S. Maxwell and J. Tirado-Rives, J. Am. Chem. Soc., 1996, 118, 11225.

26 E. K. Watkins and W. L. Jorgensen, J. Phys. Chem. A, 2001, 105, 4118 .

27 M. G. Martin and J. I. Siepmann, J. Phys. Chem. B, 1998, 102, 1569.

28 S. T. Cui, J. I. Siepmann, H. D. Cochran and P. T. Cummings, Fluid Phase Equilib., 1998, 146, 51.

29 A. A. H. Pádua, J. Deschamps, I. Marrucho, S. Sarraute and M. F. Costa Gomes, in 13th European Symposium on Fluorine Chemistry, Bordeaux, France, July 15-20, 2001.

30 Y. Miyano, Fluid Phase Equilib., 1999, 158-160, 29. 
31 W. Smith and T. R. Forester, The DL_POLY package of molecular simulation routines, version 2.12, The Council for the Central Laboratory of Research Councils, Daresbury Laboratory, Warrington, UK, 1999.

32 M. P. Allen and D. J. Tildesley, Computer Simulation of Liquids, Oxford University Press, Oxford, UK, 1987.
33 D. Frenkel and B. Smit, Understanding Molecular Simulation. From Algorithms to Applications, Academic Press, San Diego, 1996.

34 B. Widom, J. Chem. Phys., 1963, 39, 2908.

35 R. P. Bonifácio, C. McCabe, E. Filipe, M. F. Costa Gomes and A. A. H. Pádua, Mol. Phys., 2002, 100, 2547. 